\title{
The First Principle of Thermodynamics under Relativistic Conditions and Temperature
}

\author{
Emil Veitsman \\ Independent Researcher \\ Email: evveitsman@gmail.com
}

\begin{abstract}
The first principle of thermodynamics under relativistic conditions and with allowance for surface tension was formulated. With the help of this law it was shown that the temperature of the system studied had varied according to H.Ott at the adiabatic and non-adiabatic acceleration, i.e., it (the temperature) had increased for the observer in the laboratory reference frame if the velocity of the object, in turn, had increased. A.Einstein considered that the temperature had, on the contrary, to decrease under these conditions when the velocity increased. It was shown where he had made an error.
\end{abstract}

Keywords: Special relativity; relativistic thermodynamics; surface tension; temperature.

\section{Introduction}

Relativistic thermodynamics was born more than century ago - in 1907 when Kurd von Mosengeil's article had been published [1]; he was Plank's pupil. According to conclusions done by K. von Mosengeil and M.Planck, the temperature $T$ of the system under study has to vary with proportion to $\sqrt{1-\beta^{2}}$ under relativistic conditions and at adiabatic acceleration; $\beta=v / c ; v$ is the velocity of the system studied by an observer being in the reference frame at rest; $c$ is the velocity of light. A. Einstein came to the similar conclusion [2].

Studying the first principle of thermodynamics under relativistic conditions, nobody in the 20th century took into consideration the surface tension; meanwhile it is an important thermodynamic parameter, so important as pressure $p$. The both M.Planck with his pupil and A. Einstein wrote down the first principle of thermodynamics under relativistic conditions without the surface tension as well. Besides, the both great scientists made an error integrating further Gibbs' equation; we shall show it below, in section 2 .

E.V.Veitsman was the first researcher who had understood the importance of the surface tension for relativistic thermodynamics [3-5]. He showed that the surface tension is a Lorentz invariant [3]; he showed as well that the interface equations of state were correct under relativistic conditions exclusively if the temperature of system under study varied in inverse proportion to $\sqrt{1-\beta^{2}}$ [4]. E.Veitsman also obtained expressions for specific thermodynamic functions $\left(\mathrm{J} \cdot \mathrm{cm}^{-2}\right)$ under the relativistic conditions [5] (the internal energy $U$, the enthalpy $H$, the free energy $F$, and free enthalpy $G$ ). These functions are correct under the relativistic ones if $T \sim 1 / \sqrt{1-\beta^{2}}$. Thus the results obtained by Veitsman are in a full accordance with a result obtained by H.Ott for the relativistic temperature $T$, i.e., $T=T_{0} / \sqrt{1-\beta^{2}}[6]$; (here and below the symbol "0" denotes that this quantity is at rest). All above results are completely correct for the adiabatic acceleration of the system.

At last H. Callen and G. Horwitz consider that the temperature $T$ in general is a relativistic invariant [7].

M. Planck writes down the first principle of thermodynamics

$$
d U=d Q+d A
$$

as

$$
d U_{0}=d Q_{0}-p_{0} d V_{0}
$$

where $Q$ is the heat put into the system or carried off it $(\mathrm{J}) ; A$ the work done by the system or with it $(\mathrm{J})$. 
Under relativistic conditions the work $A$ is written down by M. Planck as

$$
\begin{gathered}
d A=-p d V+\mathbf{v} \cdot d \mathbf{G} \\
\mathbf{G}=\frac{U_{0}+p_{0} V_{0}}{c^{2} \sqrt{1-\beta^{2}}}
\end{gathered}
$$

where $\mathbf{v}$ is the vector of the system velocity; G the momentum, the symbol "." means vector multiplication [8].

G and

$$
\frac{i}{c}(U+p V)
$$

form the four-dimensional vector (4-vector) of energy-momentum, which has an invariant length equal to

$$
\frac{i}{c}\left(U_{0}+p_{0} V_{0}\right)
$$

$i$ is the imaginary unit.

Evidently, we can write down the first principle of thermodynamics in view of the surface tension (normal case) as

$$
d U_{0}=d Q_{0}-p_{0} d V_{0}+\sigma_{0} d \omega_{0}
$$

where $\omega_{0}$ is the element of area $\left(\mathrm{cm}^{2}\right)$.

Then the relations (3) and (4) are written down as

$$
\begin{gathered}
d A=-p d V+\sigma d \omega+\mathbf{v} \cdot d \mathbf{G} \\
\frac{U_{0}+p_{0} V_{0}-\sigma_{0} \omega_{0}}{c^{2} \sqrt{1-\beta^{2}}}
\end{gathered}
$$

And, consequently, the relations (5) and (6) should have a form

$$
\begin{gathered}
\frac{i}{c}(U+p V-\sigma \omega) \\
\frac{i}{c}\left(U_{0}+p_{0} V_{0}-\sigma_{0} \omega_{0}\right)
\end{gathered}
$$

Here we have to note that the quantity $\omega$ will transform as $v \rightarrow c$ in different ways: depending on the orientation of the surface in space (see Veitsman's articles [3, 9] and Fig.1 and 2(see Appendix)). Attempting to obtain the transformation law of the temperature as $v \rightarrow c$, M.Planck used dependences (3) and (4) but not (10) and (11). Other researchers did the same not taking account of surface tension, therefore the results obtained by them were incorrect. So the main goals of this paper are:

1.the obtaining of the first principle of thermodynamics under relativistic conditions in view of the surface tension;

2.the obtaining of the temperature transformation under these conditions from the above mentioned principle at the adiabatic and non-adiabatic acceleration.

\section{Solving the Problem}

Write down the first principle of thermodynamics (normal conditions) in view of the surface tension as

$$
d Q=d U+d E_{k i n}+p_{0} d V-\sigma d \omega-d A_{a c}-\mathbf{v} \cdot d \mathbf{G}
$$

where $E_{k i n}$ is the macrokinetic energy of the object under study; $A_{a c}$ the work expended on the system acceleration up to a velocity $v<<c ; E_{k i n}=A_{a c}$. The momentum $\mathbf{G}$ is taken here according to (9).

We will adiabatically accelerate the object up to a relativistic velocity $v$ now and will integrate (12) term by term from state 1 of the system to state 2 for the case represented in Fig.1 (see Appendix) taking into account (8), (9) and the equalities $p=p_{0}, \sigma=\sigma_{0}, \omega=\omega_{0}$; we have to note as well that here and below we do not study a whole object - only its part (a subsystem):

$$
\int_{1}^{2} d Q=\int_{1}^{2} d U+p_{0} \int_{1}^{2} d V-\sigma_{0} \int_{1}^{2} d \omega-\int_{1}^{2} v d G,
$$




$$
Q_{2}(v)-Q_{(0) 1}=U_{2}-U_{(0) 1}+p_{0} V_{2}-p_{0} V_{(0) 1}-\int_{1}^{2} v \frac{d v}{c^{2} \sqrt{\left(1-\frac{v^{2}}{c^{2}}\right)^{3}}}\left\{U_{0}+p_{0} V_{0}-\sigma_{0} \omega_{0}\right\}
$$

Now we take the integral in (14).

$$
\begin{aligned}
& \int_{1}^{2} v \frac{d v}{c^{2} \sqrt{\left(1-\frac{v^{2}}{c^{2}}\right)^{3}}}\left\{U_{0}+p_{0} V_{0}-\sigma_{0} \omega_{0}\right\}=\left\{U_{0}+p_{0} V_{0}-\sigma_{0} \omega_{9}\right\} \int_{1}^{2} \beta \frac{d \beta}{\sqrt{\left(1-\beta^{2}\right)^{3}}}= \\
& =\left.\left\{U_{0}+p_{0} V_{0}-\sigma_{0} \omega_{0}\right\} \frac{1}{\sqrt{1-\beta^{2}}}\right|_{1} ^{2}=\left\{U_{0}+p_{0} V_{0}-\sigma_{0} \omega_{0}\right\}\left(\frac{1}{\sqrt{1-\beta^{2}}}-1\right), \beta=v / c .(15)
\end{aligned}
$$

According to M. Planck [8],

in view of the surface tension we have:

$$
U=\frac{1}{\sqrt{1-\beta^{2}}}\left\{U_{0}+\beta^{2} p_{0} V_{0}\right\}
$$

$$
U=\frac{U_{0}+\beta^{2} p_{0} V_{0}+\sigma_{0} \omega_{0}\left(\sqrt{1-\beta^{2}}-1\right)}{\sqrt{1-\beta^{2}}}
$$

Then taking into consideration (16), we can write down (14) as

$$
\begin{aligned}
& Q_{2}(v)-Q_{(0) 1}=\frac{1}{\sqrt{1-\beta^{2}}}\left\{U_{0}+\beta^{2} p_{0} V_{0}+\sigma_{0} \omega_{0}\left(\sqrt{1-\beta^{2}}-1\right)\right\}-U_{0}+p_{0} V_{0} \sqrt{1-\beta^{2}}- \\
& -p_{0} V_{0}-\left\{U_{0}+p_{0} V_{0}-\sigma_{0} \omega_{0}\right\}\left(\frac{1}{\sqrt{1-\beta^{2}}}-1\right)
\end{aligned}
$$

If we do not input the heat in an accelerated system from its source, then the left side of (18) equals zero, and we cannot obtain any dependences of the kind $T=T(v)$; at first sight the dependence (18) does not contain the temperature. However, this relationship contains it - in a latent form. Show it. Let an object be, e.g., the liquid moving with a relativistic velocity v. For the observer being at rest in a laboratory reference frame the temperature of this liquid depends on the cooperative velocity $\boldsymbol{V}$ of the microparticles in the object relative to its centre of mass. Then for the researcher the velocity components of the total velocity $w$ of certain microparticle in the moving liquid equal [10]:

$$
\begin{gathered}
w_{1}=\frac{w_{1}^{\prime}+v}{1+\frac{v w_{1}^{\prime}}{c^{2}}} \\
w_{2}=\frac{w_{2}^{\prime} \sqrt{1-\beta^{2}}}{1+\frac{v w_{1}^{\prime}}{c^{2}}} \\
w_{3}=\frac{w_{3}^{\prime} \sqrt{1-\beta^{2}}}{1+\frac{v w_{1}^{\prime}}{c^{2}}}
\end{gathered}
$$

where $w_{i}^{\prime}(i=1,2,3)$ are the velocity components of the microparticle in the moving reference frame.

Taking into consideration the dependences (19-20), it is easy to understand that the heat in the moving system increases for the observer as $v \rightarrow c$, since for him the cooperative velocity of the microparticles increases in the system moving relative to its mass centre. If the heat increases, so does 
the temperature. However, $S=S_{0}$ in our case, this means $d Q=S d T$, and (15) contains, in fact, two parts. The first one is related to the internal energy, the second to the heat. Then (18) takes the form

$$
\begin{aligned}
& Q_{2}(v)-Q_{(0) 1}=\gamma\left\{U_{0}+p_{0} V_{0}-\sigma_{0} \omega_{0}\right\}\left(\frac{1}{\sqrt{1-\beta^{2}}}-1\right)= \\
& =\frac{1}{\sqrt{1-\beta^{2}}}\left\{U_{0}+\beta^{2} p_{0} V_{0}+\sigma_{0} \omega_{0}\left(\sqrt{1-\beta^{2}}-1\right)\right\}-U_{0}+p_{0} V_{0} \sqrt{1-\beta^{2}}-p_{0} V_{0}- \\
& -\alpha\left\{U_{0}+p_{0} V_{0}-\sigma_{0} \omega_{0}\right\}\left(\frac{1}{\sqrt{1-\beta^{2}}}-1\right) ; \alpha+\gamma=1 ; 0 \leq \alpha<1 .
\end{aligned}
$$

Equation (22) is completely consistently; it is correct in the range of velocities $0-\mathbf{v}$ for $Q_{2}(v)=\frac{Q_{0}}{\sqrt{1-\beta^{2}}}$. As a result, $T=T_{0} / \sqrt{1-\beta^{2}}$, i.e., in accordance to H.Ott. If it were $Q_{2}(v)=Q_{0} \sqrt{1-\beta^{2}}$, we would come to absurdity as $v \rightarrow c$. Indeed, the left side of (22) here tends to zero but the right side of $(22)$ does to $\infty$.

M.Planck as well as A. Einstein were mistaken considering that the temperature had to transform at adiabatic acceleration according to the law

$$
T=T_{0} \sqrt{1-\beta^{2}}
$$

as $v \rightarrow \infty$. The cause of this mistake will be shown below, in discussion.

Now we study the second case represented in Fig.2 (see Appendix).

The relation (14) should now be written down in view of the relation

$$
\omega=\omega_{0} \sqrt{1-\beta^{2}}
$$

as

$$
\int_{1}^{2} d Q=U_{2}-U_{(0) 1}+p_{0} V_{2}-p_{0} V_{(0) 1}-\sigma_{0} \omega_{2}+\sigma_{0} \omega_{(0) 1}-\int_{1}^{2} v \frac{d v}{c^{2} \sqrt{\left(1-\frac{v^{2}}{c^{2}}\right)^{3}}}\left\{U_{0}+p_{0} V_{0}-\sigma_{0} \omega_{0}\right\}
$$

Since instead of the ratio (17), we have now the ratio

then the formula (18) takes the form:

$$
U=\frac{U_{0}+\beta^{2}\left(p_{0} V_{0}-\sigma_{0} \omega_{0}\right)}{\sqrt{1-\beta^{2}}}
$$

taking into consideration (19-21), we have now:

$$
\begin{aligned}
& Q_{2}(v)-Q_{(0) 1}=\gamma\left\{U_{0}+p_{0} V_{0}-\sigma_{0} \omega_{0}\right\}\left(\frac{1}{\sqrt{1-\beta^{2}}}-1\right)= \\
& \frac{1}{\sqrt{1-\beta^{2}}}\left\{U_{0}+\beta^{2}\left(p_{0} V_{0}-\sigma_{0} \omega_{0}\right)\right\}-U_{0}+p_{0} V_{0} \sqrt{1-\beta^{2}}- \\
& -p_{0} V_{0}-\sigma_{0} \omega_{0} \sqrt{1-\beta^{2}}+\sigma_{0} \omega_{0}-\alpha\left\{U_{0}+p_{0} V_{0}-\sigma_{0} \omega_{0}\right\}\left(\frac{1}{\sqrt{1-\beta^{2}}}-1\right)
\end{aligned}
$$

$$
\begin{aligned}
& Q_{2}(v)=\frac{1}{\sqrt{1-\beta^{2}}}\left\{U_{0}+\beta^{2}\left(p_{0} V_{0}-\sigma_{0} \omega_{0}\right)\right\}+p_{0} V_{0} \sqrt{1-\beta^{2}}-\sigma_{0} \omega_{0} \sqrt{1-\beta^{2}}- \\
& -\alpha\left\{U_{0}+P_{0} V_{0}-\sigma_{0} \omega_{0}\right\}\left(\frac{1}{\sqrt{1-\beta^{2}}}-1\right)
\end{aligned}
$$




$$
\begin{aligned}
& Q_{2}(v)=\frac{1}{\sqrt{1-\beta^{2}}}\left\{U_{0}+\beta^{2}\left(p_{0} V_{0}-\sigma_{0} \omega_{0}\right)\right\}+p_{0} V_{0} \sqrt{1-\beta^{2}}-\sigma_{0} \omega_{0} \sqrt{1-\beta^{2}}- \\
& -\alpha\left\{U_{0}+P_{0} V_{0}-\sigma_{0} \omega_{0}\right\}\left(\frac{1}{\sqrt{1-\beta^{2}}}-1\right)
\end{aligned}
$$

The equation (29) is correct as and in case 1 , if $Q_{2}(v)=\frac{Q_{0}}{\sqrt{1-\beta^{2}}}$; it is incorrect if $Q_{2}(v)=Q_{0} \sqrt{1-\beta^{2}}$.

There are more complicated cases than ones represented in Fig.1 and 2(see Appendix), in particular, the cases of the droplet or bubble, however we do not study them in this paper.

We should here add that we come to the absurdity, if we consider that the temperature $T$ is the Lorentz-invariant.

Now determine, how the temperature and heat have to transform under relativistic conditions when the heat is input into the system from the outside. To solve this problem, we should take into consideration that the thermodynamical state of the system is independent of the way of its transition to it. Let there be two states of the system ( 1 and 2$)$ and two its intermediate ones ( 1 and $\left.2^{\prime}\right)$. In state 1 the system is at rest and contains some quantity of heat which is equal to $Q_{01}$. We input into the system an additional quantity of the heat $\Delta Q_{01}$; now the system is in the 1 . The heat in the system equals $Q_{01}+\Delta Q_{01}$. Accelerate adiabatic the system up to velocity $\boldsymbol{V}$. Now the system is in state 2 ; the heat of the system is

$$
Q_{2}=\frac{Q_{01}+\Delta Q_{01}}{\sqrt{1-\beta^{2}}}
$$

However, the system can go to state 2 otherwise: through state 2 . To do that, we must accelerate adiabatic the system being in state $1^{\prime}$ up to the velocity $\boldsymbol{v}$. Then the system will be in state 2 . Its heat equals

$$
Q_{2}=\frac{Q_{01}}{\sqrt{1-\beta^{2}}}
$$

Further, we input a quantity of heat into the system $\Delta Q_{2}$ and transfer it to state 2 . Then the quantity of heat in the system $Q_{2}^{\prime}$ equals

Evidently,

$$
Q_{2}=Q_{2}+\Delta Q_{2}=\frac{Q_{01}}{\sqrt{1-\beta^{2}}}+\Delta Q_{2}
$$

From equation (33) it follows that

$$
\frac{Q_{01}+\Delta Q_{1}}{\sqrt{1-\beta^{2}}}=\frac{Q_{01}}{\sqrt{1-\beta^{2}}}+\Delta Q_{2}
$$

$$
\frac{\Delta Q_{01}}{\sqrt{1-\beta^{2}}}=\Delta Q_{2}
$$

Now we can formulate the first principle of thermodynamics under relativistic conditions (3-D formalism):

$$
d U=d Q_{s}+d Q_{T}+d A_{d}+\mathbf{v} \cdot d \mathbf{G}_{s},
$$

where $Q_{s}$ is the heat changing the entropy of the system (entropic heat). i.e., $d Q=T d S ; Q_{T}$ the heat not changing the entropy of the system (relativistic heat), i.e., $d Q=S d T ; A_{d}$ the work of deformation, e.g., $d A_{d}=-p d V+\sigma d \omega ; \mathrm{v} \cdot d \mathrm{G}_{\mathrm{s}}$ the increment of the work expended on the increasing of microparticles velocity of the system moving relative to its mass centre minus the increment $\left(d Q_{T}\right)$ of the work transformed into heat for the observer being in the laboratory reference frame (see above).

If $\mathrm{v}=0$, the law (35) will be transformed in the law (1). 
Taking into consideration the relations $(9)-(11)$, we can write down:

$$
\begin{aligned}
& \mathbf{G}_{T}=\left\{\frac{\gamma\left(U_{0}+p_{0} V_{0}-\sigma_{0} \omega_{0}\right)}{c^{2} \sqrt{1-\beta^{2}}} v_{x} ; \frac{i \gamma(U+p V-\sigma \omega)}{c}\right\} \\
& \mathbf{G}_{s}=\left\{\frac{\alpha\left(U_{0}+p_{0} V_{0}-\sigma_{0} \omega_{0}\right)}{c^{2} \sqrt{1-\beta^{2}}} v_{x} ; \frac{i \alpha(U+p V-\sigma \omega)}{c}\right\}
\end{aligned}
$$

The quantities in braces of (36) and (37) form, in Minkowski, space vectors of energy-momentum $\left(v_{x}=v_{y}=0\right)$ having invariant lengths equal to

$$
\frac{i \zeta\left(U_{0}+p_{0} V_{0}-\sigma_{0} \omega_{0}\right)}{c} ; \zeta=\alpha, \gamma .
$$

We do not exclude the case when $\alpha=0$, then $\gamma=1$ and the ratio (34) disappears but the formulas containing $\alpha$ are simplified.

In [11] C.MØller represents a quantity $\Delta Q_{s}$ as

$$
\Delta Q=\Delta\left\{\mathbf{G}^{(h)}, \frac{i}{c} \Delta Q\right\}
$$

where $\Delta \mathbf{G}^{(\mathrm{h})}=\frac{\Delta Q}{c^{2}} v ; \Delta Q$ is here the amount of heat transferred to the system during the process; in fact. $\Delta Q=d Q_{s}$ (see above the law (35)).

The relation (39) was obtained by MØller with taking into account the influence of the vessel walls, containing (the vessel) our substance, i.e., in fact, for a system consisting of two subsystems: a substance and the vessel with its walls. It is important to note that the law (32) was obtained without the influence of the walls of the vessel.

The law (35) is correct in Euclidian space up to a velocity of the system motion $v_{\max }$. If $v>v_{\max }$, the microparticle velocities for the observer in the laboratory reference frame begin to prevail in the direction $\mathrm{X}_{1}$ over the microparticle ones in the directions $\mathrm{X}_{2}$ and $\mathrm{X}_{3}$ with accordance to the relativistic law of the velocity composition. Now both the heat and temperature are not already scalars they are vectors. Then we can write down the law (35) as

$$
d U=d Q_{k(s)} k+d Q_{k(T)} k+d A_{d}+\mathbf{v} \cdot d \mathbf{G}_{s}
$$

where $\boldsymbol{k}=\left(\begin{array}{l}1 \\ 1 \\ 1\end{array}\right)$ is the dimensionless vector; $Q_{k(s)}$ and $Q_{k(T)}$ are the vectors; $k=1,2,3$.

\section{Discussion}

As seen above, the temperature of the system is present in the first principle of thermodynamics in a latent form - by means of the second principle of thermodynamics

$$
\delta S=\frac{\delta Q}{T},
$$

where $S$ is the entropy, which is Lorentz-invariant according to Planck, i.e.,

$$
S=S_{0} \text {. }
$$

If we write down the transformation of heat $Q$ under relativistic conditions as

$$
Q=Q_{0} \sqrt{1-\beta^{2}}
$$

then the temperature has to transform under these conditions according to (23), i.e., according to M.Planck. However, if we write down the transformation of $\mathrm{Q}$ as

$$
Q=\frac{Q_{0}}{\sqrt{1-\beta^{2}}}
$$

then the temperature $\mathrm{T}$ has to transform under these conditions according to the following formula 


$$
T=\frac{T_{0}}{\sqrt{1-\beta^{2}}}
$$

i.e., according to H.Ott.

As we have seen above, relations (44) and (45) do not lead to the absurdity, relations (23) and (43), on the contrary, lead to it. Where were Planck and Einstein mistaken? We make an attempt to show it making a start from (2). Einstein proceeds from Gibb's equation taken in the form (normal state):

$$
T d S=d E+p d V-v d G,
$$

where $\mathrm{E}$ is the internal energy.

Accelerate adiabatically our system up to the velocity v. Further, integrate (43) term by term from state $1(\mathrm{v}=0)$ up to state $2(\mathrm{v} \rightarrow \infty)$ :

$$
\int_{1}^{2} T d S=\int_{1}^{2} d E+\int_{1}^{2} p d V-\int_{1}^{2} v d G
$$

Since $S_{0}=S$, then in view of (16)

$$
E_{2}-E_{1}+p_{0} V-p_{0} V_{0}-\left(U_{0}+p_{0} V_{0}\right)\left(\frac{1}{\sqrt{1-\beta^{2}}}-1\right)=0 .
$$

However, Einstein meanwhile operates with TdS, when there is not any input of heat in the system! We cannot use the above Gibbs ratio here, we are to use only the first principle of thermodynamics under condition that $d Q=S_{0} d T$ (see above)! If we input the heat in our system accelerated up to a velocity $\mathrm{v}$, then the increment of the heat $\mathrm{dQ}$ will contain the term TdS, however the heart of the matter has not to change. As before, we come to absurdity considering that the temperature will be transformed according to Planck-Einstein's law. Taking the transformation of the temperature according to H.Ott under these conditions, we have no contradictions.

The transformation of heat and temperature under relativistic conditions is considered to depend on the velocity of the source of heat coming to the system (see, e.g., van Kampen work [12]). In this regard the author of [12] examines two cases: 1 . when the object under study and the source of heat are moving with the same velocity, 2. when they have different velocities. The problem is solved in Minkowski space under some assumptions. In particular, van Kampen proceeds from the assumption that the internal energy $U$ will not transform under relativistic conditions of the system by formula (16), but as

$$
U=\frac{U_{0}}{\sqrt{1-\beta^{2}}}
$$

Then in the first case the heat had to transform in an adiabatic acceleration of the system according to (44) and the temperature had to transform according to (45) if entropy $S=S_{0}$.

In the second case the heat transforms under relativistic conditions according to complicated laws. In order to find them, van Kampen uses an imaginary model. There are two black bodies a and b separated by a thin metallic sheet. Relative to the laboratory frame, a and $\mathrm{b}$ have velocities $u^{a}$ and $u^{b}$ parallel to the sheet. The heat may be leaking from the subsystem a to the subsystem b. Of course, such a system does not exist in nature and cannot be created artifically. According to van Kampen, the heat $\mathrm{Q}$ in the subsystems a and $\mathrm{b}$ has to transform under relativistic conditions as

$$
\begin{aligned}
d Q^{a} & =-\rho_{a}+\gamma \rho_{b} \\
d Q^{b} & =-\rho_{b}+\gamma \rho_{a}
\end{aligned}
$$

where $\rho_{a}$ and $\rho_{b}$ are the energy density in the subsystems multiplied by $\frac{1}{4} \Delta A \Delta t, \quad \gamma=\left(1-u^{2}\right)^{-1 / 2} ; \Delta A$ is the area of a small hole through which the heat goes from the subsystem a to the subsystem $\mathrm{b}, \Delta t$ the interval of time when the hole is open; $\mathrm{u}=\frac{v}{C}, \mathrm{c}$ is the velocity of light adopted equal to 1 . Then we have for the whole system

$$
d Q_{a}+d Q_{b}=(\gamma-1)\left(\rho_{a}+\rho_{b}\right)>0
$$

and should note the following. Using the above model, van Kampen has obtained a complicated law of the heat transformation under relativistic conditions. 
However, using of different imaginary models, we can obtain some special relations not having any fundamental importance.

\section{Conclusions}

1.The first principle of thermodynamics was obtained under relativistic conditions in view of the surface tension.

2.Using the first principle of thermodynamics, it was shown that the temperature varied under relativistic conditions in adiabatic acceleration according to H.Ott, i.e., in inverse proportion to $\sqrt{1-\beta^{2}}$.

3.It was shown where A.Einstein made the mistake which led afterwards to the incorrect dependence $T=T_{0} \sqrt{1-\beta^{2}}$.

\section{References}

1. Mosengeil, K. von: Theory der stationaren Strahlung in einem gleichformig bewegten Hohlraum. Annalen der Phys. 22, 867-904 (1907).

2. Einstein, A.: Ueber das Relativitaetprincip und die aus demselben gezogenen Folgerungen. Jahrbuch deutsche Radioaktivitaet und Elektronik. №4, 411-462 (1907).

3. Veitsman, E.V.: On Relativistic Surface Tension. Colloid Interface Sci. 265, 174-178 (2003).

4. Veitsman, E.V.: Relativistic State Equations for the Interface. Ibid, 290, 231-234 (2005); Corrigendum, 295, 592 (2006); 333, 411 (2009).

5. Veitsman, E.V.: Specific thermodynamical Potential on Surfaces under Relativistic Conditions. Ibid, 337, 355-357 (2009).

6. Ott, H.: Lorentz Transformation der Waerme und der Temperatur. Zeitschrift f. Phys. 175, 70-104 (1963).

7. Callen, H. and Horwitz, G.: Relativistic Thermodynamics. Am. J. Phys. 39, 938- 947(1971).

8. Madelung, E.: Die matematische Hilfsmittel des Physikers. Springer-Verlag, Berlin (1957).

9. Veitsman, E. V.: Some Problems in Relativistic Thermodynamics. Journal of Experimental and Theoretical Physics, 105, 1057-1067 (2007).

10.Levich, V.G.: The Course of Theoretical Physics, V.1 (in Russian). Fizmatgiz, Moscow (1962).

11.MØller, C.: Relativistic Thermodynamics. A strange Incident in History of Physics. Det Kongelige Danske Videnskabernes SelskabMatematisk-fysiske Meddelelser. 36, 1-26 (1967). See also C. Moller's article "The Thermodynamics in the Special and the General Theory of Relativity". In: "Old and New Problems in Elementary Particles", G.Puppl, G. (eds) pp. 202-221 (1968).

12.Van Kampen, N.G.: Relativistic Thermodynamics of Moving Systems. Phys. Rev., 173, 295-301 (1968).

\section{Appendix}

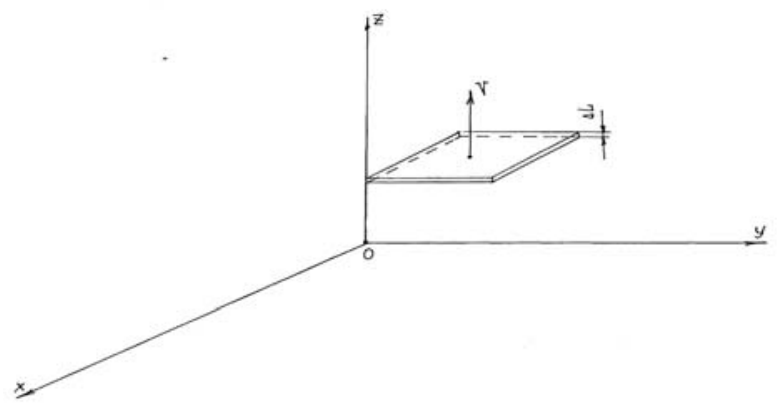

Figure 1. The moving flat interface; the velocity $\mathbf{v}$ of its motion is perpendicular to the flat one. $\Delta L$ is the thickness of the surface. 


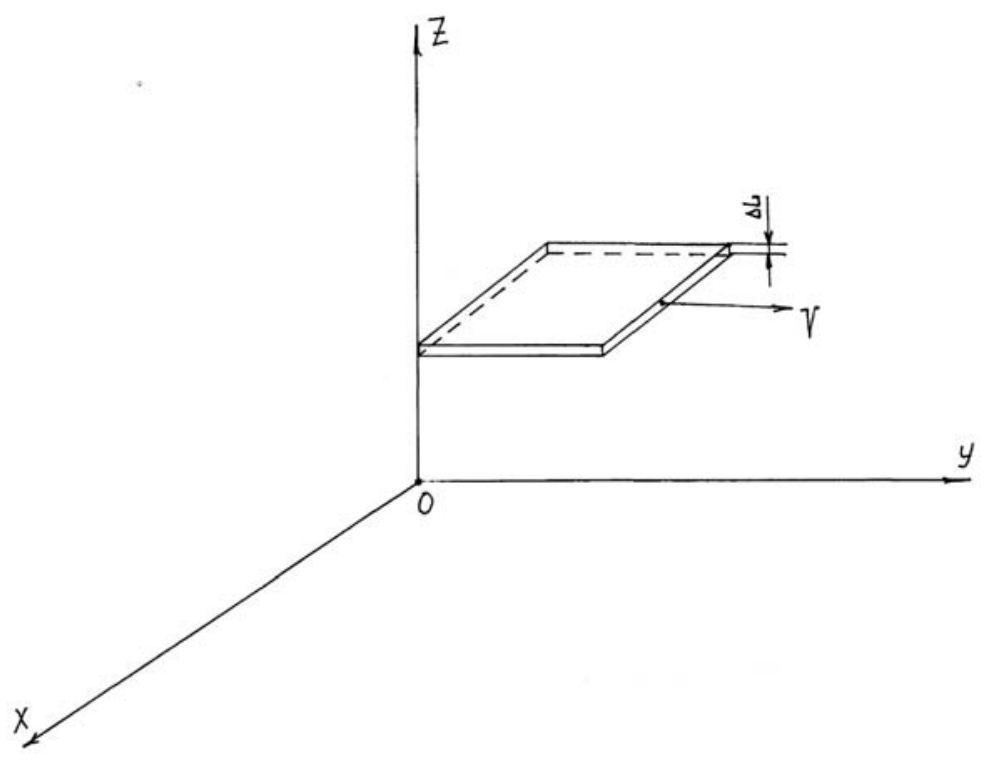

Figure 2. The moving flat interface; the velocity $\mathrm{v}$ of its motion is parallel to the flat one. $\Delta L$ is the thickness of the surface. 